\title{
The fatty acid composition of muscle and adipose tissue of steers offered unwilted or wilted grass silage supplemented with sunflower oil and fishoil
}

\author{
F. Noci ${ }^{1,2}$, F. J. Monahan ${ }^{2}$, N. D. Scollan ${ }^{3}$ and A. P. Moloney ${ }^{1} *$ \\ ${ }^{1}$ Teagasc, Grange Research Centre, Dunsany, Co. Meath, Ireland \\ ${ }^{2}$ School of Agriculture, Food Science and Veterinary Medicine, University College Dublin, Dublin 4, Ireland \\ ${ }^{3}$ Institute of Grassland and Environmental Research, Plas Goggerdan, Aberystwyth SY23 3EB, UK
}

(Received 20 February 2006 - Revised 9 October 2006 - Accepted 23 October 2006)

\begin{abstract}
The effects of the type of grass silage and dietary inclusion of fish oil (FO) on the fatty acid profile of bovine intramuscular and subcutaneous adipose tissue were investigated. Eighty Friesian steers were assigned ( $n 10)$ to unwilted or wilted silage, and to one of four rations which contained, per kg, $80 \mathrm{~g}$ of sunflower oil and either 0, 10, 20 or $40 \mathrm{~g}$ of FO replacing lard. Animals were slaughtered after $108 \mathrm{~d}$ and the fatty acid profile of the neutral, polar and total lipid fractions of the M. longissimus dorsi, and the total lipid fraction of the subcutaneous adipose tissue were determined. Wilting of grass prior to ensiling increased the concentration of conjugated linoleic acid (CLA) in intramuscular total lipid ( $P<0 \cdot 01)$, but did not affect the $n$-6:n-3 PUFA ratio. Increasing FO supply linearly increased $(P<0 \cdot 05)$ the concentration of the cis- 9 , trans-11 and trans-10,cis-12 isomers of CLA and trans-11 18:1 predominantly in the neutral lipid fraction of intramuscular total lipid, and linearly decreased the $n$-6:n-3 PUFA ratio. Wilting of grass prior to ensiling increased the concentration of CLA in subcutaneous adipose tissue $(P<0 \cdot 001)$, while increasing FO supply linearly increased the concentration of cis-9,trans-11 CLA. From a human nutrition perspective, increasing the level of FO in the ration or wilting of grass prior to ensiling appear to modify the fatty acid composition of beef muscle favourably. However, the health implications of associated increases in trans fatty acids remain to be elucidated.
\end{abstract}

Conjugated linoleic acid: Fish oil: Wilted silage: PUFA

In humans, fat of ruminant origin represents a high proportion of the dietary intake of saturated fatty acids (SFA) (Demeyer \& Doreau, 1999), which have been implicated in the onset of CVD (Department of Health, 1994). However, ruminant fats also contain higher concentrations of conjugated linoleic acid (CLA) than other food fat sources and are the main sources of CLA in the human diet (Chin et al. 1992). CLA refers to a mixture of positional and geometric isomers of linoleic acid. The cis-9,trans-11 isomer is the most common natural isomer with biological activity, representing $75-90 \%$ of total CLA in meat depending on the diet of the animal, but biological activity has been proposed for other isomers, in particular trans-10,cis-12 (Pariza et al. 2001).

The anticancer effect of CLA was initially discovered in animal models using lipid extracted from beef (Ha et al. 1987) and an anticancer effect of beef CLA has been confirmed in vitro by De la Torre et al. (2006). A range of other positive isomer-specific effects on human health has been proposed, including a reduction in atherosclerosis, decreased inflammation and improved cardiovascular health (e.g. Pariza et al. 2001). Consequently, there is considerable interest in increasing the concentration of CLA in ruminant fat.

CLA is produced following incomplete ruminal biohydrogenation of dietary 18:2 (Kepler \& Tove, 1967) and by tissue desaturation of trans-11 18:1, another product of incomplete ruminal biohydrogenation of dietary fatty acids (Griinari et al. 2000). An increase in the concentration of CLA in ruminant muscle fat has been achieved primarily by dietary addition of plant oils or seeds rich in PUFA (Enser et al. 1999; Mir et al. 2002; Raes et al. 2004; Noci et al. $2005 b$ ). Thus, supplementation with sunflower oil (SFO), a rich source of $18: 2$, has been shown to increase the concentration of CLA and trans-11, 18:1 by providing substrate for ruminal biohydrogenation (Mir et al. 2002; Noci et al. $2005 b$ ). Inclusion of fish oil (FO) has been shown to increase the concentration of CLA in milk (reviewed by Chilliard et al. 2001; Offer et al. 2001; Shingfield et al. 2003), and a combination of a source of $18: 2$ and FO increased the concentration of CLA in milk still further (AbuGhazaleh et al. 2002, 2003). The first hypothesis tested in this study was that bovine muscle and subcutaneous adipose tissue concentrations of CLA and long-chain n-3 PUFA would respond in a dose-dependent manner to supplementation of diets with FO and that FO could be used to optimise CLA accretion in ruminants fed a PUFA-rich ration.

Grazed and conserved grass are the major sources of $n$-3 PUFA, particularly $18: 3$, in ruminant diets. This makes fat from ruminants fed grass-based diets an important source

\footnotetext{
Abbreviations: CLA, conjugated linoleic acid; FO, fish oil; GC, gas chromatography; NL, neutral lipid; PL, polar lipid; SFA, saturated fatty acids; SFO, sunflower oil. * Corresponding author: Dr A. P. Moloney, fax + 353 469026154, email aidan.moloney@teagasc.ie
} 
of $n$-3 PUFA for humans. On a laboratory scale, wilting grass prior to ensiling decreased the content of total fatty acids and $18: 3 n-3$ in silage (Dewhurst \& King, 1998). While wilting grass prior to ensiling is environmentally beneficial, it may result in animals fed wilted silage having lower concentrations of $n-3$ PUFA in tissue than animals fed unwilted silage. The second hypothesis tested was that the effects of the wilting process are reflected in the fatty acid composition of muscle and subcutaneous adipose tissue of cattle consuming the resulting silage.

\section{Materials and methods}

\section{Experimental design and animal management}

Eighty Friesian steers (mean initial body weight $565 \mathrm{~kg}$, SD 37.24) were blocked on initial body weight and assigned to one of eight dietary treatments (n 10/treatment) in a randomised complete block (initial body weight) design. Animals were assigned at random to pens in a slatted-floor shed that accommodated five or six animals/pen and were fed individually through Calan gates. The initial silage allowances were $15 \mathrm{~kg}$ of wilted silage and $30 \mathrm{~kg}$ of unwilted silage, and were increased, as the experiment progressed, to 20 and $35 \mathrm{~kg}$, respectively. The silages were offered once daily, and refusals (which were rare) were recorded daily. The silages were prepared from a sward of predominantly perennial ryegrass in mid May. Grass for unwilted silage was ensiled directly with 3 litres of Addsafer (Interchem Ltd., Dublin, Ireland) (48\% formic acid and $16 \%$ ammonium tetraformate)/tonne grass. The remaining grass was wilted for an average of $32 \mathrm{~h}$ (maximum temperature $20 \cdot 2^{\circ} \mathrm{C}$, minimum temperature $5.7^{\circ} \mathrm{C}$, relative humidity $68.1 \%$ ), turned once and then ensiled without an additive. Concentrate rations were formulated to have $120 \mathrm{~g}$ fat $/ \mathrm{kg}$, which included $80 \mathrm{~g}$ of SFO/ $\mathrm{kg}$ and increasing levels of FO $(0,10,20$ or $40 \mathrm{~g} / \mathrm{kg}$ concentrate) derived from a mix of mackerel and herring oil (Fish Industries, Killybegs, Co. Donegal, Ireland), balanced with decreasing amounts of lard (Table 1). Animals were offered one of the four concentrate rations, at an initial daily allowance of $4.5 \mathrm{~kg} / \mathrm{head}$, which increased with increasing body weight to average $5 \mathrm{~kg} /$ head over the duration of the $108 \mathrm{~d}$ experiment. The concentrates were offered daily in a separate container, in two equal meals, and no refusals were observed. On the day of slaughter, animals were weighed without fasting and transported $120 \mathrm{~km}$ to a commercial facility (Meadow Meats, Rathdowney, Co. Laois, Ireland) within $3 \mathrm{~h}$ from Grange Research Centre and slaughtered within $60 \mathrm{~min}$ of arrival.

\section{Post-slaughter measurement and sampling procedure}

Carcass and perirenal fat weights were recorded immediately after slaughter. The carcasses were hung from the Achilles tendon and chilled in the abattoir for $24 \mathrm{~h}$. The M. longissimus dorsi and associated muscles and adipose tissue were excised from the right side of the carcass and transported to the Teagasc National Food Centre (Castleknock, Dublin, Ireland) where they were held at $4^{\circ} \mathrm{C}$ for a further $24 \mathrm{~h}$. The procedures for tissue sampling, lipid extraction and fatty acid analysis of the neutral lipid (NL) and polar lipid (PL) fractions
Table 1. Ingredients and formulation of the concentrate rations

\begin{tabular}{lrrrr}
\hline & \multicolumn{4}{c}{ Fish oil inclusion $(\mathrm{g} / \mathrm{kg})$} \\
\cline { 2 - 5 } Ingredients $(\mathrm{g} / \mathrm{kg})$ & 0 & 10 & 20 & 40 \\
\hline Barley & 345 & 345 & 345 & 345 \\
Sugarbeet pulp & 360 & 360 & 360 & 360 \\
Soyabean meal & 140 & 140 & 140 & 140 \\
Molasses & 10 & 10 & 10 & 10 \\
Mineral/vitamins & 25 & 25 & 25 & 25 \\
Oil & & & & \\
$\quad$ Sunflower oil & 80 & 80 & 80 & 80 \\
Lard & 40 & 30 & 20 & 0 \\
$\quad$ Fish oil & 0 & 10 & 20 & 40 \\
\hline
\end{tabular}

* The mineral and vitamin mix contained $\mathrm{Ca}(28.5 \%), \mathrm{P}(1.6 \%)$, $\mathrm{Na}(5.6 \%)$, vitamin $\mathrm{A}(150 \mathrm{mg} / \mathrm{kg})$, vitamin $D_{3}(3.1 \mathrm{mg} / \mathrm{kg})$, vitamin $E(16,7-50)$, cobalt carbonate $(42 \mathrm{mg} / \mathrm{kg})$, cupric sulphate $(500 \mathrm{mg} / \mathrm{kg})$, calcium iodate $(910 \mathrm{mg} / \mathrm{kg})$, iron sulphate $(1000 \mathrm{mg} / \mathrm{kg})$, manganese sulphate $(5800 \mathrm{mg} / \mathrm{kg})$, sodium selenite $(16 \mathrm{mg} / \mathrm{kg})$ and zinc sulphate $(7500 \mathrm{mg} / \mathrm{kg})$ on an as-fed basis.

of intramuscular fat and total lipid (TL) fraction of subcutaneous adipose tissue were as described by Noci et al. (2005a).

In brief, extracted lipid was separated into NL and PL fractions using solid-phase extraction cartridges with $500 \mathrm{mg}$ of aminopropyl packing (Bone-Elut $500 \mathrm{mg}, 3 \mathrm{ml}$ reservoir; Varian Instruments, Palo Alto, CA). The separated lipid classes were dried, weighed, dissolved in toluene and initially methylated with $\mathrm{NaOCH}_{3}$, which was followed with a $4 \%$ solution of $\mathrm{HCl}$ in methanol. Both methylation procedures were carried out at $50^{\circ} \mathrm{C}$ for $20 \mathrm{~min}$, and tricosanoic acid (C23:0) methyl ester was used as an internal standard for fatty acid quantification.

The fatty acid methylesters were separated by gas chromatography (GC) using a Varian 3800 gas chromatograph (Varian Instruments) equipped with a CP-Sil 88 capillary column $(100 \mathrm{~m} \times 0.25 \mathrm{~mm}$ i.d., $0.2 \mu \mathrm{m}$ film thickness; Chrompack, The Netherlands) and a Varian 8400 autosampler. The injector and the flame ionization detector were kept at constant temperatures of 250 and $260^{\circ} \mathrm{C}$, respectively. The column oven temperature was held at $40^{\circ} \mathrm{C}$ for $2 \mathrm{~min}$, increased at $20^{\circ} \mathrm{C} / \mathrm{min}$ to $80^{\circ} \mathrm{C}$ and held for $2 \mathrm{~min}$, increased to $160^{\circ} \mathrm{C}$ at $20^{\circ} \mathrm{C} / \mathrm{min}$, to $220^{\circ} \mathrm{C}$ at $4^{\circ} \mathrm{C} / \mathrm{min}$, and to $240^{\circ} \mathrm{C}$ at $2^{\circ} \mathrm{C} / \mathrm{min}$ and held for $8 \mathrm{~min}$. The total run time was $43 \mathrm{~min}$, and the carrier gas used was $\mathrm{H}_{2}$. For peak identification, a standard mix of 37 fatty acid methylesters (Supelco Inc., Bellefonte, PA) was used, and individual standards from Matreya (Matreya Inc., Pleasant Gap, PA) were used for identification of those fatty acid methylesters not contained in the mix.

\section{Feed chemical analysis}

The dry matter content of the concentrates and silages was determined as described by Moloney et al. (1996) and Porter \& Murray (2001), respectively. Concentrates were analysed for crude protein concentration as described by Association of Analytical Chemists (1990), for ash concentration as described by Moloney et al. (1996) and for oil Procedure B (extraction after acid hydrolysis) as described in European Communities (1984). The fatty acid composition of lipid sources and feedstuffs was determined as described by Sukhija \& Palmquist (1988). The fatty acid methylesters were 
dissolved in toluene and analysed by GC following the GC conditions described above.

\section{Statistical analysis}

Data were subjected to the analysis of variance procedures of Genstat (Release 3.2, Lawes Agricultural Trust, Rothamstead Experimental Station, UK). The model used had block, type of silage, level of FO inclusion and the interaction between the main effects as sources of variation. Linear and quadratic effects of increasing levels of inclusion of FO were partitioned using orthogonal polynomials. The concentration of fatty acids in intramuscular TL was calculated from the concentrations of fatty acids in PL and NL.

\section{Results}

\section{Feed composition}

The fatty acid compositions of the dietary fat sources are summarised in Table 2 and the chemical compositions of the silages and concentrates are summarised in Table 3. Wilted silage had a lower total fat and fatty acid content than unwilted silage mainly as a result of the lower $18: 3 n-3$ and total PUFA content. The concentrates had similar total oil, crude protein and ash contents. The concentrate containing $40 \mathrm{~g} \mathrm{FO} / \mathrm{kg}$ had the lowest SFA and MUFA contents and the highest $n-3$ PUFA and total PUFA contents.

\section{Animal production}

Concentrate intake was similar for all treatments, but consumption of wilted silage was higher than of unwilted silage, resulting in a higher total dry matter intake for the wilted silage-based rations (Table 4). Neither the type of silage nor the level of inclusion of FO in the diet affected average daily gain or pre-slaughter weight of the steers. However, the interaction between the two main effects was significant for carcass weight. Thus, when unwilted silage was offered, steers receiving 0 or $10 \mathrm{~g} \mathrm{FO} / \mathrm{kg}$ concentrate had the highest carcass weight, which decreased for steers fed $20 \mathrm{~g}$ $\mathrm{FO} / \mathrm{kg}$ concentrate, but increased again for those receiving $40 \mathrm{~g} \mathrm{FO} / \mathrm{kg}$ concentrate. With wilted silage, however, steers fed 0 or $10 \mathrm{~g} \mathrm{FO} / \mathrm{kg}$ concentrate had the lowest carcass weight, which increased when animals were fed the concentrates containing 20 and $40 \mathrm{~g} \mathrm{FO} / \mathrm{kg}$.

\section{Total intramuscular lipids}

Feeding wilted instead of unwilted silage led to an increase in the concentration of trans-9 18:1, trans-11 $18: 1(P=0 \cdot 07)$, cis-9,trans-11 CLA and trans-10,cis-12 CLA and to a decrease in the $n-6: n-3$ PUFA ratio $(P=0 \cdot 06)$ (Table 5).

Increasing the level of inclusion of FO in the concentrates led to a significant linear increase in the concentration of trans-9 18: 1 (quadratic term also significant), trans-11 18:1, trans10,cis-12 CLA, cis-9,trans-11 CLA, 20:0, 20:1, 20:2n-6, $20: 5 n-3,22: 0,22: 1,22: 2 n-6$ and $22: 6 n-3$, and to a linear decrease in the $n-6: n-3$ PUFA ratio (Table 5).

There was an interaction between the effects of type of silage and increasing levels of inclusion of FO on the total concentration of intramuscular fatty acids. As the level of inclusion of FO increased, there was an increasing quadratic response in muscle from steers offered unwilted silage, but a linear decrease in muscle from those offered wilted silage. A similar interaction was found for the concentration of $12: 0,16: 0,17: 0,17: 1,18: 0$, cis-9 $18: 1$ and $18: 3 n-3$, total SFA and MUFA.

Table 2. Fatty acid composition of oil supplements (Mean and standard deviation)

\begin{tabular}{|c|c|c|c|c|c|c|}
\hline \multirow{2}{*}{$\begin{array}{l}\text { Fatty acid composition } \\
\text { (g/100 g fatty acids) }\end{array}$} & \multicolumn{2}{|c|}{ Lard $(n 6)$} & \multicolumn{2}{|c|}{ Fish oil ( $n$ 8) } & \multicolumn{2}{|c|}{ Sunflower oil ( $n$ 8) } \\
\hline & Mean & SD & Mean & SD & Mean & SD \\
\hline $14: 0$ & $1 \cdot 17$ & 0.06 & $4 \cdot 19$ & 0.64 & 0.06 & 0.00 \\
\hline $16: 0$ & $21 \cdot 11$ & 0.38 & $10 \cdot 55$ & 0.68 & $5 \cdot 34$ & 0.11 \\
\hline $18: 0$ & $14 \cdot 30$ & 0.47 & $2 \cdot 26$ & 0.15 & 3.80 & 0.24 \\
\hline $18: 1$ & 37.72 & 0.90 & $12 \cdot 70$ & 0.53 & $26 \cdot 16$ & 0.24 \\
\hline $18: 2 n-6$ & $16 \cdot 80$ & 0.12 & 1.87 & 0.09 & 61.49 & 0.40 \\
\hline $18: 3 n-3$ & 1.56 & 0.30 & 1.57 & 0.06 & 0.16 & 0.02 \\
\hline $20: 4$ & 0.26 & 0.03 & 0.64 & 0.05 & 0.03 & 0.00 \\
\hline $20: 1$ & 0.70 & 0.62 & 10.09 & 0.48 & 0.20 & 0.00 \\
\hline $20: 2 n-6$ & 0.69 & 0.02 & 3.28 & 0.19 & 0.02 & 0.00 \\
\hline $20: 5 n-3$ & 0.03 & 0.03 & 6.95 & 0.45 & 0.01 & 0.01 \\
\hline $22: 1$ & 0.04 & 0.01 & 18.96 & $2 \cdot 10$ & 0.01 & 0.01 \\
\hline $22: 2 n-6$ & 0.03 & 0.01 & 0.98 & 0.07 & 0.00 & 0.00 \\
\hline $22: 5 n-3$ & $0 \cdot 16$ & 0.06 & 1.45 & 0.11 & 0.00 & 0.00 \\
\hline $22: 6 n-3$ & 0.07 & 0.03 & $11 \cdot 76$ & 0.60 & 0.00 & 0.00 \\
\hline SFA & 37.53 & 0.20 & 18.02 & 1.42 & 11.34 & 0.44 \\
\hline MUFA & 40.65 & 0.49 & $46 \cdot 40$ & 1.48 & 26.48 & 0.22 \\
\hline PUFA & $20 \cdot 22$ & 0.28 & 29.05 & 1.07 & 61.76 & 0.41 \\
\hline Total $n-6$ PUFA $\dagger$ & $18 \cdot 11$ & 0.17 & 7.06 & 0.19 & 61.54 & 0.39 \\
\hline Total $n-3$ PUFA & 1.81 & 0.36 & 21.95 & 1.07 & 0.19 & 0.03 \\
\hline
\end{tabular}

SFA, saturated fatty acids.

† Total $n-6$ PUFA $=$ sum of $18: 2,20: 4$ and $22: 2$.

$\ddagger$ Total $n$-3 PUFA $=$ sum of $18: 3 n-3,20: 5,22: 5$ and $22: 6$. 
Table 3. Chemical and fatty acid composition of grass silages and concentrate rations

(Mean and standard deviation)

\begin{tabular}{|c|c|c|c|c|c|c|c|c|c|c|c|c|}
\hline & & & & & \multicolumn{8}{|c|}{ Fish oil $\mathrm{g} / \mathrm{kg}$} \\
\hline & \multicolumn{2}{|c|}{ Unwilted silage ( $n$ 8) } & \multicolumn{2}{|c|}{ Wilted silage $(n 8)$} & \multicolumn{2}{|c|}{$0(n 8)$} & \multicolumn{2}{|c|}{$10(n 8)$} & \multicolumn{2}{|c|}{$20(n 8)$} & \multicolumn{2}{|c|}{$40(n 8)$} \\
\hline & Mean & SD & Mean & SD & Mean & SD & Mean & SD & Mean & SD & Mean & SD \\
\hline DM (g/kg) & 211 & 8.42 & 423 & $30 \cdot 77$ & 891 & $5 \cdot 60$ & 892 & 3.37 & 893 & 2.69 & 894 & 1.65 \\
\hline Crude protein (g/kg DM) & 184 & $10 \cdot 57$ & 184 & $7 \cdot 11$ & 156 & $6 \cdot 32$ & 160 & $11 \cdot 0$ & 154 & 6.63 & 161 & $10 \cdot 28$ \\
\hline Ash (g/kg DM) & 88.5 & $5 \cdot 62$ & $96 \cdot 6$ & 3.80 & 65 & 3.52 & 67 & $2 \cdot 20$ & 72 & 4.08 & 72 & 4.53 \\
\hline DM digestibility (g/kg) & 753 & $13 \cdot 81$ & 784 & $11 \cdot 18$ & - & - & - & - & - & - & - & - \\
\hline Oil $(A H)^{\star}(g / k g ~ D M)$ & $40 \cdot 5$ & $2 \cdot 66$ & $32 \cdot 6$ & 1.41 & 131 & $9 \cdot 33$ & 128 & $10 \cdot 91$ & 128 & $7 \cdot 63$ & 133 & $9 \cdot 46$ \\
\hline $\begin{array}{l}\text { Fatty acid composition } \\
\text { (mg fatty acid/g DM) }\end{array}$ & $(n 8)$ & & $(n 8)$ & & $(n 12)$ & & $(n 12)$ & & $(n 12)$ & & $(n 12)$ & \\
\hline $16: 0$ & $3 \cdot 27$ & 0.22 & 3.09 & 0.34 & $13 \cdot 20$ & $2 \cdot 10$ & $12 \cdot 75$ & 1.56 & 11.41 & $2 \cdot 96$ & $10 \cdot 24$ & 1.88 \\
\hline $18: 0$ & 0.32 & 0.03 & 0.31 & 0.07 & $7 \cdot 17$ & $1 \cdot 21$ & $6 \cdot 51$ & 0.86 & $5 \cdot 61$ & 1.05 & 3.88 & 0.75 \\
\hline $18: 1$ & 0.70 & 0.05 & 0.62 & 0.13 & $31 \cdot 74$ & 5.77 & 30.87 & 4.88 & 29.85 & 6.00 & $25 \cdot 25$ & $5 \cdot 16$ \\
\hline $18: 2 n-6$ & 3.49 & 0.17 & 3.42 & 0.33 & 48.93 & 10.62 & 55.90 & 7.93 & $56 \cdot 66$ & 10.82 & $51 \cdot 24$ & $10 \cdot 41$ \\
\hline $18: 3 n-3$ & $13 \cdot 86$ & 0.71 & 11.39 & 0.86 & 2.02 & 0.67 & 1.88 & 0.86 & 1.61 & 0.29 & $1 \cdot 70$ & 0.23 \\
\hline $20: 1$ & 0.04 & 0.02 & 0.01 & 0.01 & 0.22 & 0.22 & 1.04 & 0.45 & $2 \cdot 28$ & 0.66 & 3.35 & 1.47 \\
\hline $20: 4 n-6$ & - & - & - & - & 0.09 & 0.01 & 0.11 & 0.03 & 0.17 & 0.07 & 0.20 & 0.08 \\
\hline $20: 5 n-3$ & - & - & - & - & 0.06 & 0.03 & 0.55 & 0.18 & 1.27 & 0.41 & $2 \cdot 00$ & 0.93 \\
\hline $22: 5 n-3$ & - & - & - & - & 0.05 & 0.01 & 0.15 & 0.02 & 0.26 & 0.08 & 0.41 & $0 \cdot 10$ \\
\hline $22: 6 n-3$ & - & - & - & - & 0.03 & 0.02 & 0.87 & 0.18 & 1.87 & 0.60 & $3 \cdot 24$ & 0.92 \\
\hline SFA & 4.57 & 0.40 & $4 \cdot 20$ & 0.43 & 22.05 & 3.52 & $21 \cdot 29$ & 2.58 & $19 \cdot 31$ & 4.26 & 16.89 & $2 \cdot 28$ \\
\hline MUFA & 1.30 & 0.06 & 1.46 & 0.62 & 32.40 & 5.51 & 33.54 & 4.64 & 34.59 & 6.88 & 31.36 & 4.55 \\
\hline PUFA & 17.53 & 0.84 & 14.96 & 1.13 & $56 \cdot 39$ & 10.57 & 61.04 & 8.65 & 65.50 & 13.01 & 64.52 & 9.23 \\
\hline Total $n-6$ PUFA $\dagger$ & 3.68 & 0.18 & 3.57 & 0.33 & 54.55 & 10.53 & 57.94 & 8.24 & 60.49 & 11.77 & 57.55 & 7.58 \\
\hline Total $n$-3 PUFA $\ddagger$ & $13 \cdot 86$ & 0.71 & 11.39 & 0.86 & 1.83 & 0.39 & $3 \cdot 11$ & 0.48 & 5.01 & $1 \cdot 31$ & 6.97 & 1.94 \\
\hline Total fatty acids & $26 \cdot 30$ & 1.32 & $24 \cdot 12$ & 1.41 & 114.0 & 19.35 & $118 \cdot 9$ & $15 \cdot 81$ & $121 \cdot 0$ & 24.12 & $116 \cdot 0$ & $2 \cdot 60$ \\
\hline
\end{tabular}

DM, dry matter; SFA, saturated fatty acids.

*Ether extract after acid hydrolysis

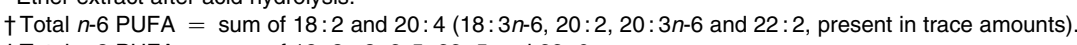

total $n$-3 PUFA $=$ sum of $18: 3 n-3,2 ; 5,22: 5$ and $22: 6$. 


\section{Intramuscular neutral lipids}

Feeding wilted instead of unwilted silage lead to an increase in the proportion of trans-9 18:1, cis-9,trans-11 CLA and trans10,cis-12 CLA and MUFA, and to a decrease in the proportions of 18: $0(P=0.07)$, SFA $(P=0.05)$ and $n-6$ PUFA $(P=0.06)$ (Table 6).

Increasing the level of inclusion of FO in the concentrates led to a significant linear increase in the proportion of trans$1118: 1$, cis-9,trans-11 CLA, 18:3n-6, 20:0, 20:1, 22:0, $22: 1$ and $22: 2 n-6$, and to a linear decrease in the proportion of cis-9 18:1, 18:2n-6, 18:3n-3 (quadratic term also significant), 20:3n-6, MUFA $(P=0 \cdot 07)$ and $n-6$ PUFA and in the $n-6: n-3$ PUFA ratio (Table 6).

There was an interaction between the effects of type of silage and increasing level of FO inclusion on the proportion of 20:4n-6 which decreased following a quadratic trend as the level of FO increased when unwilted silage was fed, but was unchanged when wilted silage was offered.

\section{Intramuscular polar lipids}

Feeding wilted instead of unwilted silage led to a decrease in the proportion of $20: 4 n-6$ in muscle PL (Table 7). Increasing the level of inclusion of FO in the concentrates led to a significant linear increase in the proportion of $17: 1,20: 1,20: 5 n-3$, $22: 2 n-6,22: 6 n-3$ and $n-3$ PUFA and to a linear decrease in the proportion of $20: 4 n-6$, SFA (quadratic) and n-6 PUFA and the $n-6: n-3$ PUFA ratio (Table 7).

\section{Total subcutaneous adipose tissue lipids}

Feeding wilted instead of unwilted silage led to an increase in the concentration of cis-9,trans-11 CLA and total PUFA and in the PUFA:SFA (P:S) ratio, and to a decrease in the concentration of 18:0 $(P=0 \cdot 07)$ (Table 8$)$.

Increasing the level of inclusion of FO in the concentrates led to a significant linear increase in the concentration of cis-9 18: $1(P=0.06)$, cis-9, trans-11 CLA, 20:1, 20:0 $(P=0 \cdot 07), 20: 5 n-3 \quad(P=0 \cdot 06)$ and $22: 6 n-3$, and a linear decrease in the concentration of $18: 2 n-6,18: 3 n-3$ and total $n-6$ PUFA.

\section{Discussion}

The myriad putative health benefits of CLA, and in particular the cis-9,trans-11 isomer, have stimulated interest in increasing its concentration in beef. Since CLA is formed either directly or indirectly during ruminal biohydrogenation of dietary lipids, the most common strategy examined has been to increase/modify the supply of lipids for cattle. Recent studies have indicated an influence of dietary long-chain PUFA on pathways of biohydrogenation in the rumen, either by direct action of the PUFA or via the action of intermediate products of PUFA biohydrogenation (Scollan et al. 2001b; Shingfield et al. 2003). Specifically, these studies showed that dietary long-chain PUFA increased trans-11 18:1 as a product of rumen biohydrogenation of dietary PUFA. An increase in the outflow of trans-11 18:1 from the rumen may then induce an increase in the concentration of CLA in tissues, via the action of $\Delta^{9}$-desaturase (Griinari et al. 2000). 
Table 5. Fatty acid concentration in the total lipid fraction of intramuscular fat from M. longissimus dorsi

\begin{tabular}{|c|c|c|c|c|c|c|c|c|c|c|c|c|}
\hline \multirow{2}{*}{$\begin{array}{l}\text { Silage (S) } \\
\text { Fish oil (FO g/kg) }\end{array}$} & \multicolumn{4}{|c|}{ Unwilted } & \multicolumn{4}{|c|}{ Wilted } & \multirow[b]{2}{*}{ SED } & \multirow[b]{2}{*}{$\mathrm{S}$} & \multirow[b]{2}{*}{ FO } & \multirow[b]{2}{*}{$\mathrm{S} \times \mathrm{FO}$} \\
\hline & 0 & 10 & 20 & 40 & 0 & 10 & 20 & 40 & & & & \\
\hline \multicolumn{13}{|c|}{ Fatty acids (mg/100 g 5muscle) } \\
\hline $10: 0$ & $7 \cdot 30$ & 7.08 & 6.04 & $5 \cdot 75$ & $6 \cdot 37$ & 6.76 & $7 \cdot 22$ & $6 \cdot 60$ & $1 \cdot 131$ & NS & NS & NS \\
\hline $11: 0$ & 0.01 & 0.00 & 0.02 & 0.03 & 0.00 & 0.01 & 0.00 & 0.02 & 0.015 & NS & NS & NS \\
\hline $12: 0$ & $2 \cdot 30$ & 1.38 & $2 \cdot 03$ & 3.18 & 2.42 & 2.85 & 1.96 & 1.84 & 0.579 & NS & NS & *L \\
\hline $13: 0$ & $0 \cdot 10$ & 0.07 & 0.16 & 0.26 & $0 \cdot 10$ & 0.20 & 0.07 & $0 \cdot 10$ & $0 \cdot 108$ & NS & NS & NS \\
\hline $14: 0$ & $347 \cdot 1$ & $276 \cdot 1$ & $321 \cdot 7$ & $368 \cdot 1$ & $340 \cdot 3$ & $350 \cdot 3$ & $381 \cdot 0$ & $323 \cdot 8$ & $51 \cdot 14$ & NS & NS & NS \\
\hline $14: 1$ & $16 \cdot 15$ & $10 \cdot 90$ & 14.63 & 21.02 & $16 \cdot 21$ & $27 \cdot 17$ & $17 \cdot 76$ & $11 \cdot 11$ & 6.397 & NS & NS & * \\
\hline $15: 0$ & 34.72 & 24.63 & 31.61 & $43 \cdot 28$ & 35.66 & $37 \cdot 23$ & $35 \cdot 29$ & $32 \cdot 32$ & $5 \cdot 239$ & NS & NS & ${ }^{*} \mathrm{~L}$ \\
\hline $15: 1$ & 0.08 & 0.03 & $0 \cdot 16$ & 0.59 & 0.19 & 0.12 & 0.39 & 0.03 & 0.252 & NS & NS & NS \\
\hline $16: 0$ & 1519 & 1245 & 1366 & 1983 & 1708 & 1695 & 1599 & 1522 & $206 \cdot 5$ & NS & NS & ${ }^{* *} \mathrm{~L}$ \\
\hline $16: 1$ & $189 \cdot 8$ & $128 \cdot 2$ & $177 \cdot 4$ & $210 \cdot 4$ & $193 \cdot 6$ & $226 \cdot 8$ & $204 \cdot 3$ & $166 \cdot 5$ & 38.45 & NS & NS & NS \\
\hline $17: 0$ & $102 \cdot 1$ & $80 \cdot 77$ & 89.91 & 118.5 & $112 \cdot 2$ & $106 \cdot 9$ & $100 \cdot 5$ & 98.48 & $12 \cdot 17$ & NS & ${ }^{\star} \mathrm{Q}$ & ${ }^{*} \mathrm{~L}$ \\
\hline $17: 1$ & 38.63 & $27 \cdot 36$ & 36.52 & $46 \cdot 30$ & $46 \cdot 70$ & $46 \cdot 26$ & 39.74 & 36.88 & $6 \cdot 617$ & NS & NS & ${ }^{*} \mathrm{~L}$ \\
\hline $18: 0$ & $899 \cdot 1$ & $809 \cdot 4$ & $792 \cdot 0$ & 1121 & 1057 & 921.5 & $846 \cdot 2$ & $885 \cdot 7$ & $116 \cdot 3$ & NS & NS & NS \\
\hline $18: 1$ cis -9 & 2442 & 1719 & 2284 & 2670 & 2622 & 2725 & 2456 & 2217 & 346.5 & NS & NS & ${ }^{*} \mathrm{~L}$ \\
\hline $18: 1$ trans -9 & 14.42 & $11 \cdot 17$ & 11.09 & 22.57 & 18.88 & 18.72 & 18.78 & 20.01 & 3.534 & * & ${ }^{*} \mathrm{~L}, \mathrm{Q}$ & NS \\
\hline $18: 1$ cis-11 & $20 \cdot 59$ & $1 \cdot 10$ & 3.90 & $9 \cdot 34$ & 11.64 & $16 \cdot 50$ & 24.48 & $5 \cdot 69$ & 11.62 & NS & NS & NS \\
\hline $18: 1$ trans- 11 & $298 \cdot 8$ & $223 \cdot 6$ & $276 \cdot 9$ & $374 \cdot 7$ & $296 \cdot 8$ & $321 \cdot 1$ & 334.4 & $362 \cdot 3$ & 39.54 & 0.07 & ${ }^{* *} \mathrm{~L}$ & NS \\
\hline C18: $2 n-6$ cis & $152 \cdot 7$ & $119 \cdot 1$ & $142 \cdot 8$ & 155.5 & $163 \cdot 7$ & $160 \cdot 4$ & 165.9 & $130 \cdot 4$ & $18 \cdot 42$ & NS & NS & NS \\
\hline C18:2n-6 trans & 1.67 & 2.02 & 0.79 & $2 \cdot 41$ & 1.27 & 1.28 & 0.48 & $1 \cdot 11$ & 0.835 & NS & NS & NS \\
\hline CLA cis-9,trans- 11 & 41.88 & 32.01 & 40.38 & 59.15 & 49.56 & 53.31 & $52 \cdot 23$ & $55 \cdot 77$ & 6.785 & $\star \star *$ & ${ }^{* *} \mathrm{~L}$ & NS \\
\hline CLA trans-10,cis-12 & 1.91 & 1.33 & 1.96 & $2 \cdot 70$ & $2 \cdot 60$ & 2.55 & $2 \cdot 83$ & 3.46 & 0.718 & * & ${ }^{*} \mathrm{~L}$ & NS \\
\hline $18: 3 n-6$ & $2 \cdot 15$ & 1.96 & 2.83 & $5 \cdot 75$ & 1.95 & $3 \cdot 13$ & $2 \cdot 88$ & $2 \cdot 91$ & 1.001 & NS & ${ }^{\star *} \mathrm{~L}$ & ${ }^{*} \mathrm{~L}$ \\
\hline $18: 3 n-3$ & $28 \cdot 16$ & $22 \cdot 04$ & 25.65 & 33.55 & $31 \cdot 13$ & $30 \cdot 17$ & $29 \cdot 83$ & $26 \cdot 15$ & 3.387 & NS & NS & ${ }^{*} \mathrm{~L}$ \\
\hline $20: 0$ & $5 \cdot 64$ & $5 \cdot 62$ & $7 \cdot 26$ & 11.76 & $8 \cdot 14$ & $6 \cdot 88$ & $6 \cdot 35$ & 9.04 & 1.618 & NS & ${ }^{*} \mathrm{~L}, \mathrm{Q}$ & NS \\
\hline $20: 1$ & $14 \cdot 20$ & 11.06 & 19.71 & 37.42 & $15 \cdot 87$ & $1 \cdot 18$ & 23.40 & 32.56 & $4 \cdot 222$ & NS & ${ }^{\star \star \star \star} \mathrm{L}$ & NS \\
\hline $20: 2 n-6$ & 2.45 & 1.82 & 3.04 & 4.79 & 2.92 & 2.58 & 3.08 & 3.22 & 0.995 & NS & ${ }^{*} \mathrm{~L}$ & NS \\
\hline $20: 3 n-3$ & 0.43 & 0.29 & 0.46 & 0.74 & 0.30 & 0.41 & 0.90 & 0.42 & 0.380 & NS & NS & NS \\
\hline $20: 3 n-6$ & $10 \cdot 10$ & $7 \cdot 27$ & 7.85 & 8.79 & $10 \cdot 90$ & $10 \cdot 40$ & 8.52 & $8 \cdot 23$ & 1.761 & NS & NS & NS \\
\hline $20: 4 n-6$ & $40 \cdot 96$ & $29 \cdot 37$ & 34.34 & 22.37 & $27 \cdot 46$ & $35 \cdot 00$ & $19 \cdot 52$ & 22.49 & $10 \cdot 36$ & NS & NS & NS \\
\hline $20: 5 n-3$ & 8.43 & $7 \cdot 80$ & $10 \cdot 18$ & 14.66 & 7.69 & $10 \cdot 69$ & $9 \cdot 37$ & $12 \cdot 60$ & $2 \cdot 131$ & NS & ${ }^{* \star *} \mathrm{~L}$ & NS \\
\hline $22: 0$ & 0.71 & 0.60 & 1.32 & $1 \cdot 82$ & 0.66 & 0.54 & 0.66 & 1.33 & 0.390 & NS & ${ }^{\star \star \star} \mathrm{L}$ & NS \\
\hline $22: 1 n-9$ & $4 \cdot 12$ & 4.68 & 9.69 & $18 \cdot 31$ & $2 \cdot 31$ & $6 \cdot 22$ & $10 \cdot 38$ & $16 \cdot 29$ & 2.487 & NS & ${ }^{* * *} \mathrm{~L}$ & NS \\
\hline $22: 2 n 6$ & 4.35 & 3.54 & 6.00 & $11 \cdot 12$ & $3 \cdot 39$ & 4.70 & $5 \cdot 72$ & 9.58 & 1.309 & NS & ${ }^{\star * \star \star} \mathrm{L}$ & NS \\
\hline $22: 5 n-3$ & $16 \cdot 30$ & 12.49 & 15.00 & 18.44 & $16 \cdot 30$ & 18.56 & 14.86 & $18 \cdot 19$ & 2.637 & NS & NS & NS \\
\hline $22: 6 n-3$ & $3 \cdot 16$ & 1.79 & 3.46 & 4.78 & 2.09 & $2 \cdot 23$ & $2 \cdot 61$ & 3.84 & 0.833 & NS & ${ }^{* * *} \mathrm{~L}$ & NS \\
\hline $24: 0$ & 0.24 & 0.20 & 0.18 & 0.31 & 0.38 & 0.19 & $0 \cdot 11$ & $0 \cdot 15$ & 0.123 & NS & NS & NS \\
\hline $24: 1$ & 0.02 & 0.00 & 0.06 & 0.32 & 0.02 & 0.00 & 0.02 & 0.25 & 0.108 & NS & ${ }^{* \star *} \mathrm{~L}$ & NS \\
\hline SFA & 2918 & 2451 & 2618 & 3657 & 3272 & 3128 & 2978 & 2881 & $351 \cdot 7$ & NS & NS & ${ }^{*} \mathrm{~L}$ \\
\hline MUFA & 3039 & 2137 & 2834 & 3411 & 3225 & 3407 & 3129 & 2869 & $425 \cdot 8$ & NS & NS & ${ }^{*} \mathrm{~L}$ \\
\hline PUFA & $314 \cdot 6$ & $242 \cdot 8$ & 294.7 & $344 \cdot 8$ & $321 \cdot 3$ & 335.4 & $318 \cdot 7$ & 298.3 & 39.00 & NS & NS & NS \\
\hline $\mathrm{P}: \mathrm{S}$ ratio & 0.11 & $0 \cdot 10$ & 0.11 & 0.10 & $0 \cdot 10$ & 0.11 & 0.11 & $0 \cdot 10$ & 0.008 & NS & NS & NS \\
\hline$n-6$ fatty acids $\dagger$ & 214.4 & $165 \cdot 0$ & $197 \cdot 7$ & $210 \cdot 8$ & $211 \cdot 7$ & $217 \cdot 4$ & $206 \cdot 1$ & $177 \cdot 9$ & $26 \cdot 42$ & NS & NS & NS \\
\hline$n-3$ fatty acids $\ddagger$ & 56.48 & 44.41 & 54.75 & $72 \cdot 17$ & 57.51 & 62.06 & 57.57 & $61 \cdot 20$ & $7 \cdot 615$ & NS & ${ }^{*} \mathrm{~L}$ & NS \\
\hline n-6:n-3 ratio & 3.89 & 3.77 & 3.64 & 2.95 & 3.72 & 3.45 & 3.62 & 2.95 & 0.206 & 0.06 & ${ }^{* * *} \mathrm{~L}$ & NS \\
\hline Total fatty acids & 6521 & 5045 & 6009 & 7802 & 7078 & 7131 & 6713 & 6331 & $809 \cdot 3$ & NS & NS & *L \\
\hline
\end{tabular}

CLA, conjugated linoleic acid; SFA, saturated fatty acids; $L$ and $Q$, significant $(P<0.05)$ linear and quadratic effects of level of inclusion of fish oil; P:S, PUFA:SFA.

*** and ${ }^{* * *}$ refer to significance levels $P<0.05, P<0.01$ and $P<0.001$, respectively.

$\dagger \mathrm{n}-6$ fatty acids $=$ sum of $18: 2,18: 3 n-6,20: 2,20: 3 n-6,20: 4$ and $22: 2$

$\ddagger n-3$ fatty acids $=$ sum of $18: 3 n-3,20: 3 n-3,20: 5,22: 5$ and $22: 6$. 
Table 6. The proportion of individual fatty acids in the neutral lipid fraction of intramuscular fat from $M$. longissimus dorsi

\begin{tabular}{|c|c|c|c|c|c|c|c|c|c|c|c|c|}
\hline \multirow{2}{*}{$\begin{array}{l}\text { Silage (S) } \\
\text { Fish oil (FO g/kg) }\end{array}$} & \multicolumn{4}{|c|}{ Unwilted } & \multicolumn{4}{|c|}{ Wilted } & \multirow[b]{2}{*}{ SED } & \multirow[b]{2}{*}{$\mathrm{S}$} & \multirow[b]{2}{*}{$\mathrm{FO}$} & \multirow[b]{2}{*}{$\mathrm{S} \times \mathrm{FO}$} \\
\hline & 0 & 10 & 20 & 40 & 0 & 10 & 20 & 40 & & & & \\
\hline \multicolumn{13}{|c|}{ Fatty acids (proportion $\times 100$ ) } \\
\hline $10: 0$ & 0.13 & 0.16 & 0.11 & 0.09 & $0 \cdot 10$ & 0.11 & 0.12 & 0.12 & 0.021 & NS & NS & ${ }^{*} \mathrm{~L}$ \\
\hline $12: 0$ & 0.04 & 0.03 & 0.04 & 0.04 & 0.04 & 0.04 & 0.03 & 0.03 & 0.006 & NS & NS & NS \\
\hline $13: 0$ & - & - & - & - & - & - & - & - & - & - & - & - \\
\hline $14: 0$ & 5.85 & 6.04 & $5 \cdot 80$ & $5 \cdot 41$ & 5.08 & $5 \cdot 28$ & $6 \cdot 16$ & $5 \cdot 79$ & 0.717 & NS & NS & NS \\
\hline $14: 1$ & 0.25 & 0.23 & 0.24 & 0.27 & 0.24 & 0.38 & 0.29 & 0.21 & 0.073 & NS & NS & NS \\
\hline $15: 0$ & 0.58 & 0.53 & 0.56 & 0.61 & 0.56 & 0.57 & 0.58 & 0.58 & 0.039 & NS & NS & NS \\
\hline $15: 1$ & - & - & - & - & - & - & - & - & - & - & - & - \\
\hline $16: 0$ & 23.50 & $24 \cdot 66$ & $23 \cdot 27$ & 25.41 & 23.69 & 24.04 & 23.79 & $24 \cdot 14$ & 0.874 & NS & NS & NS \\
\hline $16: 1$ & 3.00 & $2 \cdot 66$ & 3.05 & $2 \cdot 75$ & $2 \cdot 86$ & $3 \cdot 23$ & $3 \cdot 24$ & $2 \cdot 82$ & 0.299 & NS & NS & NS \\
\hline $17: 0$ & $1 \cdot 37$ & 1.41 & $1 \cdot 35$ & 1.39 & $1 \cdot 38$ & $1 \cdot 35$ & $1 \cdot 33$ & $1 \cdot 36$ & 0.036 & NS & NS & NS \\
\hline $17: 1$ & 0.59 & 0.52 & 0.60 & 0.56 & 0.66 & 0.62 & 0.57 & 0.56 & 0.046 & NS & NS & NS \\
\hline $18: 0$ & $14 \cdot 54$ & $17 \cdot 02$ & $14 \cdot 47$ & $15 \cdot 20$ & $15 \cdot 16$ & 14.09 & $13 \cdot 16$ & $14 \cdot 44$ & $1 \cdot 174$ & 0.07 & NS & NS \\
\hline $18: 1$ cis-9 & 37.97 & $34 \cdot 73$ & $38 \cdot 40$ & 34.40 & 38.50 & 38.58 & $37 \cdot 34$ & 35.58 & 1.347 & NS & ${ }^{\star *} \mathrm{~L}$ & NS \\
\hline $18: 1$ trans-9 & 0.24 & 0.25 & $0 \cdot 21$ & $0 \cdot 31$ & 0.30 & 0.29 & 0.30 & 0.34 & 0.049 & * & NS & NS \\
\hline $18: 1$ cis- 11 & 0.27 & 0.03 & 0.08 & 0.10 & 0.14 & 0.23 & 0.44 & 0.12 & 0.177 & NS & NS & NS \\
\hline $18: 1$ trans -11 & 4.99 & 4.75 & 4.79 & $5 \cdot 21$ & 4.65 & 4.95 & $5 \cdot 35$ & $6 \cdot 11$ & 0.471 & NS & ${ }^{* *} \mathrm{~L}$ & NS \\
\hline $18: 2 n-6$ cis & $1 \cdot 33$ & $1 \cdot 18$ & $1 \cdot 16$ & 1.00 & $1 \cdot 27$ & 1.04 & $1 \cdot 16$ & 1.04 & 0.077 & NS & ${ }^{\star * \star} L$ & NS \\
\hline $18: 2 n-6$ trans & 0.02 & 0.04 & 0.01 & 0.04 & 0.02 & 0.02 & 0.01 & 0.02 & 0.014 & NS & NS & NS \\
\hline CLA cis-9, trans-11 & 0.66 & 0.65 & 0.66 & 0.78 & 0.73 & 0.78 & 0.80 & 0.91 & 0.057 & $\star \star \star *$ & ${ }^{* \star *} \mathrm{~L}$ & NS \\
\hline CLA trans-10, cis-12 & 0.03 & 0.03 & 0.03 & 0.04 & 0.04 & 0.04 & 0.05 & 0.06 & 0.010 & * & NS & NS \\
\hline $18: 3 n-6$ & 0.03 & 0.04 & 0.05 & 0.08 & 0.03 & 0.05 & 0.05 & 0.05 & 0.015 & NS & ${ }^{\star *} \mathrm{~L}$ & NS \\
\hline $18: 3 n-3$ & 0.34 & 0.31 & 0.29 & 0.30 & 0.34 & 0.29 & 0.30 & 0.28 & 0.021 & NS & ${ }^{\star \star} \mathrm{LQ}$ & NS \\
\hline $20: 0$ & 0.08 & 0.09 & 0.12 & 0.14 & 0.10 & 0.08 & 0.08 & 0.14 & 0.018 & NS & ${ }^{\star \star \star} \mathrm{L}$ & NS \\
\hline $20: 1$ & 0.23 & 0.25 & 0.35 & 0.52 & 0.25 & 0.28 & 0.39 & 0.54 & 0.047 & NS & ${ }^{\star \star \star} \mathrm{L}$ & NS \\
\hline $20: 2 n-6$ & 0.04 & 0.03 & 0.04 & 0.05 & 0.04 & 0.02 & 0.04 & 0.04 & 0.013 & NS & NS & NS \\
\hline $20: 3 n-3$ & 0.01 & 0.01 & 0.01 & 0.01 & 0.00 & 0.01 & 0.01 & 0.01 & 0.006 & NS & NS & NS \\
\hline $20: 3 n-6$ & 0.03 & 0.03 & 0.02 & 0.02 & 0.03 & 0.03 & 0.02 & 0.01 & 0.007 & NS & ${ }^{\star \star \star} \mathrm{L}$ & NS \\
\hline $20: 4 n-6$ & 0.06 & 0.10 & 0.06 & 0.05 & 0.06 & 0.02 & 0.06 & 0.08 & 0.014 & NS & NS & $\star \star \star *$ \\
\hline $20: 5 n-3$ & 0.00 & 0.00 & 0.00 & 0.00 & 0.01 & 0.00 & 0.00 & 0.00 & 0.002 & NS & NS & NS \\
\hline $22: 0$ & 0.01 & 0.01 & 0.03 & 0.02 & 0.01 & 0.01 & 0.01 & 0.02 & 0.006 & NS & ${ }^{\star *} \mathrm{~L}$ & NS \\
\hline $22: 1 n-9$ & 0.05 & 0.07 & 0.12 & 0.23 & 0.02 & 0.05 & 0.14 & 0.27 & 0.034 & NS & ${ }^{\star \star \star} \mathrm{L}$ & NS \\
\hline $22: 2 n-6$ & 0.03 & 0.03 & 0.04 & 0.05 & 0.02 & 0.02 & 0.03 & 0.05 & 0.010 & NS & ${ }^{\star \star *} \mathrm{~L}$ & NS \\
\hline $22: 5 n-3$ & 0.06 & 0.06 & 0.06 & 0.06 & 0.06 & 0.06 & 0.06 & 0.07 & 0.009 & NS & NS & NS \\
\hline $22: 6 n-3$ & 0.00 & 0.00 & 0.00 & 0.01 & 0.00 & 0.00 & 0.00 & 0.01 & 0.003 & NS & NS & NS \\
\hline $24: 0$ & 0.00 & 0.00 & 0.00 & 0.00 & 0.01 & 0.00 & 0.00 & 0.00 & 0.002 & NS & NS & NS \\
\hline $24: 1$ & - & - & - & - & - & - & - & - & - & - & - & - \\
\hline Total SFA & $46 \cdot 12$ & $49 \cdot 97$ & $45 \cdot 76$ & $48 \cdot 33$ & $46 \cdot 13$ & $45 \cdot 59$ & $45 \cdot 28$ & $46 \cdot 63$ & 1.645 & 0.05 & NS & NS \\
\hline Total MUFA & $47 \cdot 58$ & 43.49 & $47 \cdot 84$ & $44 \cdot 36$ & 47.63 & $48 \cdot 61$ & 48.07 & $46 \cdot 57$ & 1.517 & * & 0.07 & 0.07 \\
\hline Total PUFA & $2 \cdot 65$ & 2.50 & $2 \cdot 43$ & 2.48 & 2.65 & $2 \cdot 38$ & $2 \cdot 60$ & $2 \cdot 63$ & 0.130 & NS & NS & NS \\
\hline$n-6$ fatty acids $\dagger$ & 1.54 & 1.45 & $1 \cdot 37$ & 1.29 & 1.47 & $1 \cdot 21$ & $1 \cdot 38$ & 1.29 & 0.084 & 0.06 & ${ }^{\star *} \mathrm{~L}$ & 0.07 \\
\hline$n-3$ fatty acids $\ddagger$ & 0.41 & 0.38 & 0.36 & 0.38 & 0.40 & 0.35 & 0.37 & 0.37 & 0.027 & NS & 0.09 & NS \\
\hline$n-6: n-3$ ratio & 4.00 & 3.69 & 3.58 & $2 \cdot 71$ & 3.78 & 3.49 & 3.59 & $2 \cdot 74$ & 0.277 & NS & ${ }^{\star \star \star} \mathrm{L}$ & NS \\
\hline
\end{tabular}

CLA, conjugated linoleic acid; SFA, saturated fatty acids; $L$ and $Q$, significant $(P<0.05)$ linear and quadratic effects of level of inclusion of fish oil.

*, ** and *** refer to significance levels $P<0.05, P<0.01$ and $P<0.001$, respectively.

$\dagger n-6$ fatty acids $=$ sum of $18: 2,18: 3 n-6,20: 2,20: 3 n-6,20: 4$ and $22: 2$

$\ddagger n-3$ fatty acids $=$ sum of $18: 3 n-3,20: 3 n-3,20: 5,22: 5$ and $22: 6$.

The demonstration that trans-11 18:1 is also converted to CLA in humans and augments the CLA potential of ruminant products (Kuhnt et al. 2006) has focused attention on increasing the concentration of this fatty acid per se. The strategy used in this experiment was, first, to supply significant amounts of $18: 2 n-6$ to the rumen by feeding SFO, because increased dietary $18: 2 n-6$ per se has been shown to increase the concentration of CLA in intramuscular fat (Mir et al. 2002; Noci et al. 2005b). Secondly, in contrast to other studies in which one concentration of FO was examined (Enser et al. 1999; Shingfield et al. 2003), increasing levels of dietary FO were used to determine the response pattern of an increase in CLA and in the nutritionally important $n-6: n-3$ PUFA ratio.

With the objective of enhancing the nutritional attributes of bovine muscle fat and subcutaneous adipose tissue, the contribution of forage to the diet is important because the lipids of grass-based forages are rich in $18: 3 n-3$. Since grass silage is the major feed ingredient in housed cattle rations in Western Europe, the concentrates were offered in a conserved grass-based ration.

\section{Effects of type of silage}

Dewhurst \& King (1998) found that wilting perennial ryegrass before ensiling decreased the total fatty acid concentration, particularly the $18: 3 n-3$ concentration. A similar observation was made by Boufaïed et al. (2003), who reported a significant decrease in fatty acids of increasing chain length from 14:0 to $18: 3 n-3$ when wilted grass was compared with fresh grass. The lower concentrations of $16: 0,18: 0,18: 1,18: 2$, $18: 3 n-3$ and total fatty acids in wilted silage in the present 
Table 7. The proportion of individual fatty acids in the polar lipid faction of intramuscular fat from M. longissimus dorsi

\begin{tabular}{|c|c|c|c|c|c|c|c|c|c|c|c|c|}
\hline \multirow{2}{*}{$\begin{array}{l}\text { Silage (S) } \\
\text { Fish oil (FO g/kg) }\end{array}$} & \multicolumn{4}{|c|}{ Unwilted } & \multicolumn{4}{|c|}{ Wilted } & \multirow[b]{2}{*}{ SED } & \multirow[b]{2}{*}{$S$} & \multirow[b]{2}{*}{$\mathrm{FO}$} & \multirow[b]{2}{*}{$\mathrm{S} \times \mathrm{FO}$} \\
\hline & 0 & 10 & 20 & 40 & 0 & 10 & 20 & 40 & & & & \\
\hline \multicolumn{13}{|c|}{ Fatty acids (proportion $\times 100$ ) } \\
\hline $14: 0$ & 1.59 & 1.06 & 0.90 & 0.91 & 2.01 & 1.05 & 1.06 & 1.47 & 0.380 & NS & ${ }^{*} \mathrm{Q}$ & NS \\
\hline $14: 1$ & 0.00 & 0.00 & 0.00 & 0.01 & 0.01 & 0.02 & 0.00 & 0.00 & 0.008 & NS & NS & NS \\
\hline $16: 0$ & 23.47 & 22.49 & 22.98 & 24.54 & 25.46 & $22 \cdot 78$ & 24.36 & $23 \cdot 14$ & 1.542 & NS & NS & NS \\
\hline $16: 1$ & 1.11 & 1.15 & 0.95 & 1.35 & 1.00 & 1.14 & 1.15 & 1.12 & 0.174 & NS & NS & NS \\
\hline $17: 0$ & 3.59 & 3.49 & $3 \cdot 14$ & $2 \cdot 82$ & 3.52 & $3 \cdot 10$ & 3.06 & 3.50 & 0.447 & NS & NS & NS \\
\hline $17: 1$ & 0.44 & 0.62 & 0.59 & 0.90 & 0.61 & 0.71 & 0.68 & 0.79 & 0.144 & NS & $* * \mathrm{~L}$ & NS \\
\hline $18: 0$ & 9.59 & $7 \cdot 19$ & $7 \cdot 15$ & $6 \cdot 83$ & 11.72 & $7 \cdot 62$ & $7 \cdot 34$ & $8 \cdot 71$ & 2.023 & NS & ${ }^{*} \mathrm{Q}$ & NS \\
\hline $18: 1$ cis-9 & $25 \cdot 07$ & $26 \cdot 82$ & $26 \cdot 96$ & $29 \cdot 20$ & 23.45 & $26 \cdot 94$ & 27.83 & $25 \cdot 80$ & 2.423 & NS & NS & NS \\
\hline 18: 1 trans-9 & 0.04 & 0.02 & 0.03 & 0.02 & 0.01 & 0.02 & 0.02 & 0.02 & 0.024 & NS & NS & NS \\
\hline $18: 1$ cis -11 & 0.30 & 0.00 & 0.00 & 0.25 & 0.26 & 0.01 & 0.17 & 0.00 & 0.252 & NS & NS & NS \\
\hline 18: 1 trans- 11 & 1.68 & 1.51 & 1.56 & 1.97 & 1.31 & 1.59 & 1.60 & 1.77 & 0.229 & NS & NS & NS \\
\hline $18: 2 n-6$ cis & $13 \cdot 19$ & $14 \cdot 20$ & 13.95 & 11.93 & 13.42 & 13.95 & $15 \cdot 15$ & $12 \cdot 32$ & 1.584 & NS & NS & NS \\
\hline $18: 2 n-6$ trans & 0.01 & 0.02 & 0.03 & 0.01 & 0.00 & 0.00 & 0.01 & 0.02 & 0.015 & NS & NS & NS \\
\hline CLA cis-9, trans- 11 & 0.51 & 0.50 & 0.52 & 0.55 & 0.50 & 0.54 & 0.56 & 0.61 & 0.070 & NS & NS & NS \\
\hline CLA trans-10, cis- 12 & 0.00 & 0.00 & 0.02 & 0.00 & 0.01 & 0.00 & 0.00 & 0.01 & 0.006 & NS & NS & NS \\
\hline $18: 3 n-6$ & 0.02 & 0.05 & 0.02 & 0.03 & 0.03 & 0.05 & 0.01 & 0.03 & 0.025 & NS & NS & NS \\
\hline $18: 3 n-3$ & 1.46 & $1 \cdot 76$ & 1.68 & $1 \cdot 70$ & 1.53 & 1.76 & 1.88 & 1.73 & 0.192 & NS & NS & NS \\
\hline $20: 0$ & 0.12 & 0.32 & 0.21 & 0.22 & 0.24 & 0.24 & 0.21 & 0.18 & 0.079 & NS & NS & NS \\
\hline $20: 1$ & 0.01 & 0.01 & 0.02 & 0.06 & 0.00 & 0.00 & 0.01 & 0.09 & 0.032 & NS & $* \star \star L$ & NS \\
\hline $20: 2 n-6$ & 0.05 & 0.15 & $0 \cdot 10$ & 0.13 & 0.07 & 0.14 & 0.11 & 0.13 & 0.041 & NS & * & NS \\
\hline $20: 3 n-3$ & 0.00 & 0.00 & 0.00 & 0.00 & 0.01 & 0.00 & 0.00 & 0.00 & 0.009 & NS & NS & NS \\
\hline $20: 3 n-6$ & 1.35 & 1.27 & $1 \cdot 14$ & 1.03 & 1.37 & 1.27 & $1 \cdot 14$ & 1.30 & 0.202 & NS & NS & NS \\
\hline $20: 4 n-6$ & $6 \cdot 22$ & $5 \cdot 27$ & 5.73 & 2.64 & 3.68 & 4.90 & 2.48 & $3 \cdot 11$ & 1.409 & * & ${ }^{*} \mathrm{~L}$ & NS \\
\hline $20: 5 n-3$ & 1.36 & 1.67 & 1.75 & 2.00 & $1 \cdot 12$ & 1.62 & 1.51 & $2 \cdot 22$ & 0.286 & NS & ${ }^{* \star \star} \mathrm{L}$ & NS \\
\hline $22: 0$ & - & - & - & - & - & - & - & - & - & - & - & - \\
\hline $22: 1 n-9$ & 0.22 & 0.37 & 0.49 & 0.31 & 0.22 & 0.48 & 0.33 & 0.17 & 0.192 & NS & NS & NS \\
\hline $22: 2 n-6$ & 0.38 & 0.50 & 0.60 & 1.04 & 0.32 & 0.51 & 0.59 & $1 \cdot 16$ & 0.129 & NS & ${ }^{\star \star \star \star} \mathrm{L}$ & NS \\
\hline $22: 5 n-3$ & 2.04 & $2 \cdot 16$ & $2 \cdot 14$ & 2.02 & 2.02 & 2.33 & 1.82 & 2.44 & 0.328 & NS & NS & NS \\
\hline $22: 6 n-3$ & 0.49 & 0.37 & 0.56 & 0.60 & 0.30 & 0.32 & 0.41 & 0.60 & 0.116 & NS & ${ }^{* *} \mathrm{~L}$ & NS \\
\hline $24: 0$ & 0.00 & 0.01 & 0.00 & 0.00 & 0.00 & 0.00 & 0.00 & 0.00 & 0.005 & NS & NS & NS \\
\hline $24: 1$ & - & - & - & - & - & - & - & - & - & - & - & - \\
\hline SFA & 38.38 & 34.56 & 34.39 & 35.33 & 42.95 & 34.80 & 36.04 & 37.01 & 3.147 & NS & ${ }^{\star} Q$ & NS \\
\hline MUFA & 28.87 & 30.49 & 30.59 & 34.11 & 26.88 & 30.90 & 31.85 & 29.76 & 2.581 & NS & NS & NS \\
\hline PUFA & 27.09 & 27.93 & 28.23 & 23.68 & 24.40 & 27.41 & 25.68 & 25.68 & $2 \cdot 417$ & NS & NS & NS \\
\hline$n-6$ fatty acidst & 21.22 & 21.46 & 21.56 & $16 \cdot 80$ & 18.90 & $20 \cdot 82$ & 19.50 & 18.06 & 1.973 & NS & ${ }^{*} \mathrm{~L}$ & NS \\
\hline$n-3$ fatty acids $\ddagger$ & $5 \cdot 35$ & 5.96 & $6 \cdot 12$ & $6 \cdot 32$ & 4.99 & 6.04 & 5.62 & 7.00 & 0.642 & NS & ${ }^{* *} \mathrm{~L}$ & NS \\
\hline$n-6: n-3$ ratio & 4.00 & 3.69 & 3.58 & $2 \cdot 71$ & 3.78 & 3.49 & 3.59 & 2.74 & 0.277 & NS & 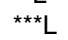 & NS \\
\hline
\end{tabular}

CLA, conjugated linoleic acid; SFA, saturated fatty acids; $L$ and $Q$, significant $(P<0.05)$ linear and quadratic effects of level of inclusion of fish oil.

${ }^{*},{ }^{* *}$ and ${ }^{* * *}$ refer to significance levels $P<0.05, P<0.01$ and $P<0.001$, respectively.

$\dagger n-6$ fatty acids $=$ sum of $18: 2,18: 3 n-6,20: 2,20: 3 n-6,20: 4$ and $22: 2$

$\ddagger n-3$ fatty acids $=$ sum of $18: 3 n-3,20: 3 n-3,20: 5,22: 5$ and $22: 6$.

study confirm the loss of fatty acids (mainly $18: 3 n-3$ ) due to the wilting process, possibly due to oxidative loss, as suggested by Dewhurst \& King (1998).

While there was an interaction between type of silage and level of FO inclusion for the concentration of total and several individual fatty acids in the NL (data not shown) and TL of muscle, the fact that the interaction was generally not detected when data were analysed as proportions of the total fatty acids confirmed that the interaction was mainly due to the small differences in fatness across the eight treatments.

With respect to the silages, daily consumption of $18: 3 n-3$ averaged $95 \mathrm{~g}$ for steers fed unwilted silage compared with the $83 \mathrm{~g}$ for steers fed wilted silage. Ruminal biohydrogenation of dietary $18: 3 n-3$ apparently eliminated this difference in intake since the proportions of intramuscular $18: 3 n-3$ were $0 \cdot 37$ and 0.36 for unwilted and wilted silage-fed steers, respectively, similar to the proportion reported by Scollan et al. (2001a) for cattle fed grass silage and concentrates.

The increase in the concentration of cis-9,trans-11 CLA in both TL and subcutaneous adipose tissue as a result of feeding wilted silage indicates a possible effect of the type of silage on rumen biohydrogenation pathways. Since the muscle concentration of CLA is linked to the outflow of trans-11 18: 1 from the rumen (Griinari et al. 2000), the results suggest that the conditions created by feeding wilted silage instead of unwilted silage were more effective in modifying microbial flora action towards an incomplete biohydrogenation of the dietary PUFA. The higher proportion of trans-9 18:1 and trans-11 18:1 in the NL (and TL) lends support to the hypothesis of an increased outflow of trans-11 18:1 as a precursor for desaturation in the muscle. An effect of concentration and type of substrate, dilution rate and rumen $\mathrm{pH}$ on the production of long-chain fatty acids, including trans $18: 1$ isomers, by mixed ruminal bacteria in continuous culture was shown by Martin \& Jenkins (2002).

\section{Effect of level of FO inclusion}

The muscle and subcutaneous adipose tissue fatty acid data in the present study generally support the findings of Enser et al. (1999) and Scollan et al. (2001a) with regard to FO 
Table 8. Fatty acid concentration in subcutaneous adipose tissue

\begin{tabular}{|c|c|c|c|c|c|c|c|c|c|c|c|c|}
\hline \multirow{2}{*}{$\begin{array}{l}\text { Silage }(\mathrm{S}) \\
\text { Fish oil (FO g/kg) }\end{array}$} & \multicolumn{4}{|c|}{ Unwilted } & \multicolumn{4}{|c|}{ Wilted } & \multirow[b]{2}{*}{ SED } & \multirow[b]{2}{*}{$\mathrm{S}$} & \multirow[b]{2}{*}{$\mathrm{FO}$} & \multirow[b]{2}{*}{$\mathrm{S} \times \mathrm{FC}$} \\
\hline & 0 & 10 & 20 & 40 & 0 & 10 & 20 & 40 & & & & \\
\hline \multicolumn{13}{|c|}{ Fatty acids (mg/g sample) } \\
\hline $10: 0$ & 0.27 & 0.27 & 0.20 & 0.22 & 0.20 & 0.22 & 0.19 & 0.25 & 0.054 & NS & NS & NS \\
\hline $12: 0$ & 0.15 & 0.15 & $0 \cdot 14$ & 0.15 & 0.12 & 0.12 & 0.13 & 0.15 & 0.030 & NS & NS & NS \\
\hline $13: 0$ & 0.03 & 0.05 & 0.03 & 0.07 & 0.03 & 0.03 & 0.03 & 0.03 & 0.018 & * & NS & NS \\
\hline $14: 0$ & 20.60 & 20.85 & 19.82 & 19.96 & $19 \cdot 11$ & 18.86 & 18.96 & $19 \cdot 28$ & 2.051 & NS & NS & NS \\
\hline $14: 1$ & 4.01 & 3.77 & $4 \cdot 15$ & 3.99 & $4 \cdot 13$ & 4.65 & 3.83 & $4 \cdot 10$ & 0.585 & NS & NS & NS \\
\hline $15: 0$ & 4.55 & $4 \cdot 30$ & 4.05 & 4.55 & 4.34 & 4.96 & $4 \cdot 17$ & 4.27 & 0.523 & NS & NS & NS \\
\hline $15: 1$ & 0.06 & 0.08 & 0.08 & 0.13 & 0.07 & 0.06 & 0.08 & 0.07 & 0.026 & NS & NS & NS \\
\hline $16: 0$ & $119 \cdot 3$ & 113.6 & $108 \cdot 0$ & $105 \cdot 6$ & $102 \cdot 0$ & $115 \cdot 3$ & $104 \cdot 1$ & $109 \cdot 7$ & $7 \cdot 736$ & NS & NS & NS \\
\hline $16: 1$ & 28.38 & $27 \cdot 23$ & $27 \cdot 27$ & $26 \cdot 70$ & $29 \cdot 35$ & 33.31 & $28 \cdot 26$ & $27 \cdot 72$ & 3.250 & NS & NS & NS \\
\hline $17: 0$ & 8.96 & 7.43 & 8.28 & $7 \cdot 50$ & $7 \cdot 25$ & $7 \cdot 76$ & $7 \cdot 15$ & 8.08 & 1.005 & NS & NS & NS \\
\hline $17: 1$ & $5 \cdot 68$ & $5 \cdot 20$ & 5.34 & $5 \cdot 61$ & $5 \cdot 68$ & 5.93 & $5 \cdot 27$ & $5 \cdot 18$ & 0.463 & NS & NS & NS \\
\hline $18: 0$ & $52 \cdot 24$ & $55 \cdot 18$ & $45 \cdot 20$ & $45 \cdot 26$ & 42.58 & $48 \cdot 36$ & 43.79 & 44.80 & 4.983 & 0.07 & NS & NS \\
\hline $18: 1$ cis-9 & $221 \cdot 3$ & $217 \cdot 3$ & $209 \cdot 3$ & $202 \cdot 7$ & $208 \cdot 3$ & $230 \cdot 5$ & $197 \cdot 8$ & $202 \cdot 2$ & $16 \cdot 23$ & NS & $0.06 \mathrm{~L}$ & NS \\
\hline $18: 1$ trans -9 & 1.70 & 0.90 & 2.93 & 3.43 & 1073 & 1.32 & 2.42 & 1046 & 1.430 & NS & NS & NS \\
\hline $18: 1$ cis- 11 & 2.03 & $2 \cdot 73$ & $3 \cdot 23$ & 4.43 & $2 \cdot 87$ & 1.68 & $2 \cdot 36$ & 5.08 & 1.879 & NS & NS & NS \\
\hline $18: 1$ trans- 11 & 30.72 & 28.40 & 24.68 & 27.45 & 20.54 & 23.97 & 24.98 & $32 \cdot 74$ & 4.672 & NS & NS & NS \\
\hline $18: 2 n-6$ cis & 6.79 & 5.96 & $5 \cdot 84$ & $5 \cdot 61$ & $7 \cdot 13$ & $6 \cdot 23$ & $6 \cdot 13$ & 5.63 & 0.512 & NS & ${ }^{\star \star \star} \mathrm{L}$ & NS \\
\hline $18: 2 n-6$ trans & 0.44 & 0.17 & 0.21 & 0.19 & 0.78 & $0 \cdot 12$ & 0.35 & 0.22 & 0.201 & NS & ** & NS \\
\hline CLA cis-9, trans- 11 & 5.47 & 6.00 & 5.48 & $6 \cdot 78$ & $6 \cdot 37$ & $7 \cdot 34$ & $6 \cdot 62$ & 8.67 & 0.756 & 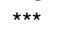 & ${ }^{* *} \mathrm{~L}$ & NS \\
\hline CLA trans -10, cis- 12 & 0.33 & 0.20 & $0 \cdot 16$ & 0.23 & 0.23 & 0.32 & 0.25 & 0.26 & 0.068 & NS & NS & NS \\
\hline $18: 3 n-6$ & 0.10 & 0.09 & 0.09 & 0.10 & 0.10 & 0.10 & 0.08 & 0.09 & 0.021 & NS & NS & NS \\
\hline $18: 3 n-3$ & 1.44 & 1.23 & $1 \cdot 10$ & 1.30 & 1.45 & 1.23 & 1.29 & $1 \cdot 12$ & 0.145 & NS & $0.05 \mathrm{~L}$ & NS \\
\hline $20: 0$ & 0.46 & 0.50 & 0.52 & 0.66 & 0.52 & 0.36 & 0.86 & 0.66 & 0.162 & NS & $0.07 \mathrm{~L}$ & NS \\
\hline $20: 1$ & 1.88 & 2.03 & $2 \cdot 25$ & 3.32 & 1.73 & $2 \cdot 10$ & $2 \cdot 82$ & 3.70 & 0.385 & NS & ${ }^{* \star \star} \mathrm{L}$ & NS \\
\hline $20: 2 n-6$ & 0.35 & 0.26 & 0.33 & 0.53 & 0.32 & 0.32 & 0.50 & 0.42 & $0 \cdot 111$ & NS & NS & NS \\
\hline $20: 3 n-3$ & 0.00 & 0.01 & 0.00 & 0.00 & 0.00 & 0.01 & 0.04 & 0.03 & 0.024 & NS & NS & NS \\
\hline $20: 3 n-6$ & 0.19 & 0.18 & 0.12 & $0 \cdot 16$ & $0 \cdot 19$ & $0 \cdot 16$ & 0.15 & 0.17 & 0.037 & NS & NS & NS \\
\hline $20: 4 n-6$ & 0.26 & 0.25 & 0.23 & 0.24 & 0.19 & 0.20 & 0.26 & 0.29 & 0.072 & NS & NS & NS \\
\hline $20: 5 n-3$ & 0.04 & 0.02 & 0.04 & 0.06 & 0.03 & 0.03 & 0.02 & 0.04 & 0.012 & NS & $0.06 \mathrm{~L}$ & NS \\
\hline $22: 0$ & 0.13 & 0.08 & 0.11 & 0.12 & $0 \cdot 12$ & $0 \cdot 11$ & $0 \cdot 15$ & 0.13 & 0.041 & NS & NS & NS \\
\hline $22: 1 n-9$ & 0.08 & 0.09 & 0.11 & 0.23 & 0.09 & $0 \cdot 19$ & 0.32 & 0.32 & 0.109 & NS & $0.07 \mathrm{~L}$ & NS \\
\hline $22: 2 n-6$ & 0.25 & 0.20 & 0.27 & 0.44 & 0.14 & 0.42 & 0.23 & 0.35 & 0.085 & NS & ${ }^{* * \mathrm{~L}}$ & * \\
\hline $22: 5 n-3$ & 0.33 & 0.20 & 0.21 & 0.22 & 0.14 & 0.24 & 0.19 & 0.25 & 0.060 & NS & NS & ${ }^{*} \mathrm{~L}$ \\
\hline $22: 6 n-3$ & 0.01 & 0.01 & 0.02 & 0.03 & 0.01 & 0.00 & 0.01 & 0.02 & 0.011 & NS & ${ }^{* *} \mathrm{~L}$ & NS \\
\hline $24: 0$ & 0.03 & 0.02 & 0.03 & 0.02 & 0.02 & 0.02 & 0.04 & 0.02 & 0.014 & NS & NS & NS \\
\hline $24: 1$ & 0.01 & 0.00 & 0.01 & 0.02 & 0.00 & 0.01 & 0.00 & 0.02 & 0.006 & NS & ${ }^{* \star \star} \mathrm{L}$ & NS \\
\hline SFA & $206 \cdot 7$ & 202.5 & $186 \cdot 4$ & $184 \cdot 1$ & $176 \cdot 3$ & $196 \cdot 1$ & 179.6 & 187.4 & $13 \cdot 50$ & NS & NS & NS \\
\hline MUFA & 295.9 & 287.7 & $279 \cdot 3$ & 278.0 & 274.5 & 303.7 & 268.1 & 282.6 & 16.54 & NS & NS & NS \\
\hline PUFA & 16.00 & 14.78 & $14 \cdot 11$ & 15.90 & $17 \cdot 07$ & $16 \cdot 72$ & $16 \cdot 13$ & 17.57 & 1.455 & * & NS & NS \\
\hline $\mathrm{P}: \mathrm{S}$ ratio & 0.08 & 0.08 & 0.08 & 0.09 & $0 \cdot 10$ & 0.09 & 0.09 & 0.09 & 0.010 & * & NS & NS \\
\hline$n-6$ fatty acids $t$ & 8.38 & $7 \cdot 11$ & $7 \cdot 10$ & $7 \cdot 27$ & 8.86 & 7.55 & $7 \cdot 70$ & $7 \cdot 17$ & 0.706 & NS & ${ }^{*} \mathrm{~L}$ & NS \\
\hline$n-3$ fatty acids $\ddagger$ & 1.82 & 1.47 & 1.37 & 1.62 & 1.62 & 1.51 & 1.55 & 1.46 & 0.175 & NS & NS & NS \\
\hline$n-6: n-3$ ratio & 4.89 & 4.94 & $5 \cdot 30$ & 4.61 & 5.58 & $5 \cdot 26$ & 4.99 & 5.04 & 0.477 & NS & NS & NS \\
\hline Total fatty acids & 546.9 & $536 \cdot 3$ & $508 \cdot 1$ & $509 \cdot 1$ & $497 \cdot 3$ & 549.0 & $492 \cdot 9$ & $521 \cdot 2$ & $25 \cdot 24$ & NS & NS & NS \\
\hline
\end{tabular}

CLA, conjugated linoleic acid; SFA, saturated fatty acids; $L$ and $Q$, significant $(P<0.05)$ linear and quadratic effects of level of inclusion of fish oil; $P: S$, PUFA:SFA.

${ }^{*},{ }^{* *}$ and ${ }^{* \star *}$ refer to significance levels $P<0.05, P<0.01$ and $P<0.001$, respectively.

† $n-6$ fatty acids $=$ sum of $18: 2,18: 3 n-6,20: 2,20: 3 n-6,20: 4$ and $22: 2$

$\ddagger n-3$ fatty acids $=$ sum of $18: 3 n-3,20: 3 n-3,20: 5,22: 5$ and $22: 6$.

supplementation of beef cattle. Of particular interest from a human nutrition perspective is the increase in cis-9,trans-11 CLA (and its precursor trans-11 18:1), 20:5n-3 and $22: 6 n$ 3 proportions in muscle and subcutaneous adipose tissue, and the decrease in the $n-6: n-3$ PUFA ratio in muscle with FO consumption. It has been established that dietary FO increases production of trans-11 18:1 in the rumen, and subsequently its concentration in milk fat or in the intramuscular adipose tissue (Chilliard et al. 2001; Scollan et al. 2001a, b). The results of the present study indicate that this effect of FO, via the supply of long-chain PUFA to the rumen (or via intermediates of their biohydrogenation in the rumen), is dosedependent. This was confirmed in a parallel study in which the concentrates containing 0,10 or $40 \mathrm{~g} \mathrm{FO} / \mathrm{kg}$ were offered to duodenally fistulated steers (Lee et al. 2005), i.e. a linear increase in the outflow of trans-11 18:1 from the rumen was observed. In contrast, there was a quadratic increase in cis-9,trans-11 CLA outflow from the rumen such that the ratio trans-11 18:1:cis-9,trans-11 CLA was greater for the $40 \mathrm{~g} \mathrm{FO} / \mathrm{kg}$ concentrate than for the $10 \mathrm{~g} \mathrm{FO} / \mathrm{kg}$ concentrate. Despite this, the trans-11 18:1:cis-9,trans-11 CLA ratio in muscle and subcutaneous adipose tissue was not influenced by FO inclusion. These data suggest a greater efficiency of absorption of cis-9,trans-11 CLA and/or a lower efficiency of absorption of trans-11 18:1 from the intestine of cattle fed the high FO concentrates or differences in metabolism 
post-absorption. The lack of an effect of FO inclusion on the trans-11 18: 1:cis-9,trans-11 CLA ratio does not support the hypothesis that long-chain PUFA inhibit tissue desaturase activity, at least at the concentration of long-chain PUFA observed in the present study. While there were analytical differences in the present study and that of Lee et al. (2005), the influence of FO or long-chain PUFA consumption per se on post-ruminal trans-11 18:1 and cis-9,trans-11 CLA transformations merit investigation.

Enser et al. (1999) reported a cis-9,trans-11 CLA concentration of $24.3 \mathrm{mg} / 100 \mathrm{~g}$ muscle from Charolais steers fed a $20 \mathrm{~g} \mathrm{FO} / \mathrm{kg}$ concentrate diet compared with an average of $57.5 \mathrm{~g} / 100 \mathrm{~g}$ muscle for cattle offered the $40 \mathrm{~g}$ FO $/ \mathrm{kg}$ concentrate in the present study. The higher concentration of this CLA isomer achieved in the present experiment may be attributed to the 3-fold and 1-5-fold higher daily intake of $18: 2 n-6$ and $18: 3 n-3$, respectively (steers consuming the $40 \mathrm{~g} \mathrm{FO} / \mathrm{kg}$ concentrate had a total intake from silage and concentrate of $227 \mathrm{~g}$ of $18: 2 n-6$ and $89 \mathrm{~g}$ of $18: 3 n-3)$, leading to higher trans-11 1 8:1 (and CLA) production through biohydrogenation (Lee et al. 2005). It should be noted that the animals in the present experiment had a higher concentration of total fatty acids in the muscle than those reported by Enser et al. (1999). Since CLA was found predominantly in the NL fraction, which increases as animals accrete lipid, this will account for some of the difference in CLA concentrations between the studies.

While earlier in vitro and laboratory animal studies convincingly demonstrated an anticancer effect of the cis-9,trans-11 isomer of CLA, this effect has not been demonstrated in humans. This relates at least in part to the aetiology of cancer and the difficulty in conducting studies on the prevention of cancer in humans. Long-term studies with appropriate end points such as incidence of cancer remain to be conducted so an anticancer effect of CLA in humans cannot be excluded. As a range of other positive isomer-specific effects on human health have been proposed, including a reduction in atherosclerosis, decreased inflammation and improved cardiovascular health (e.g. Pariza et al. 2001), the efficacy of CLA in the treatment and prevention of several of these conditions has also been examined. The findings from human studies to date have been equivocal (reviewed by Yaqoob et al. 2006). This review suggests that much of the variability in the human studies conducted to date is due to the use of mixtures of CLA isomers (e.g. trans-10,cis-12 CLA appears to have a negative effect on blood lipids while the cis-9,trans-11 isomer does not) and short duration, to which could be added insufficient statistical power to detect differences of biological significance. Considerably more information is required in this regard.

Epidemiological associations between the risk of coronary heart disease and the consumption of trans PUFA has also focused attention on the concentration of these fatty acids in food. The trans fatty acid profile in ruminant fat tends be enriched with the trans-11 isomer of $18: 1$, as was seen in the present study. Industrially derived oils such as hydrogenated vegetable oils, in contrast, have a broader spectrum of trans 18:1 isomers and have a considerably higher concentration of trans-9 and trans-10 18:1 isomers (Scollan et al. 2006). The relative risk to human health of consuming the individual isomers remains to be elucidated, but current epidemiological evidence suggests that consumption of ruminally derived trans PUFA is not a risk factor for heart disease (Jakobsen, 2006).

Data in the literature are unclear with respect to ruminal biohydrogenation of long-chain PUFA of FO. In an in vitro study, Gulati et al. (1999) suggested that the ability of rumen micro-organisms to hydrogenate long-chain PUFA is dependent on the concentration of those fatty acids in the rumen environment. Scollan et al. (2001b) reported biohydrogenation of 92 and $91 \%$ for $20: 5 n-3$ and $22: 6 n-3$, respectively, in beef cattle fed $30 \mathrm{~g} \mathrm{FO} / \mathrm{kg}$ dry matter intake. In our parallel study, Lee et al. (2005) reported corresponding values of 79 and $80 \%$ for beef cattle fed the $40 \mathrm{~g} \mathrm{FO} / \mathrm{kg}$ concentrate. In the present study, the incorporation of long-chain PUFA was relatively low in the NL fraction and in the subcutaneous adipose tissue, in agreement with Ashes et al. (1992) and Mitchell et al. (1991), respectively, while the greatest effect of dietary treatments was observed in the PL where PUFA are present in higher concentrations. While the highest level of inclusion of FO resulted in the highest incorporation of long chain $n$-3 PUFA in the muscle PL, the proportion of long-chain PUFA was lower than that found by Scollan et al. (2001a). Differences could be attributed to the different proportions of long-chain $n$-3 PUFA in the FO used, to the different proportion of oil included in the diets used (1.9 and $2.4 \%$ of dietary dry matter in our study and that of Scollan et al. (2001a), respectively) and to the different levels of fatness of the animals in the two experiments. This may also relate to the higher $18: 2 n-6$ intake in the present study as $n-6$ PUFA compete for deposition with the $n-3$ PUFA and are preferentially deposited.

In the present study, a decrease in $20: 4 n-6$ in the PL fraction with a concomitant increase in long-chain n-3 PUFA suggests that the latter were incorporated at the expense of the former to maintain the structural integrity of the membrane phospholipid fraction. Such a decrease in long-chain $n-6$ PUFA in the PL has also been observed in other studies with both ruminants and non-ruminants in which fishmeal and FO were included as dietary supplements (Morgan et al. 1992; Mandell et al. 1997).

The considerable proportion of $20: 1$ and $22: 1$ contained in the FO used in this experiment (Table 2) could explain the linear increase in very-long-chain MUFA and SFA in the NL fraction. Shingfield et al. (2003) also found increased amounts of some long-chain fatty acids $(20: 1,20: 2,22: 0$ and $22: 1)$ entering the omasal canal and being incorporated into milk fat following the inclusion of FO in the diet. Overall, however, the addition of FO had virtually no impact on the total SFA, MUFA or PUFA content of the intramuscular adipose tissue or of the subcutaneous adipose tissue. Consequently, the P:S ratio was similar across the dietary treatments and it was also considerably lower than the recommended value of 0.45 suggested by the Department of Health (1994). Our results are consistent with those of Mandell et al. (1997), Choi et al. (2000) and Scollan et al. (2001a). Scollan et al. (2003) suggested the existence of a negative exponential relationship between the amount of intramuscular fat and the P:S ratio. When animals have intramuscular fat concentrations ranging between 2000 and $4000 \mathrm{mg} / 10$ $0 \mathrm{~g}, \mathrm{P}: \mathrm{S}$ ratio values, calculated as $(18: 2 n-6+18: 3 n-3) /$ $(\Sigma(12: 0,14: 0,16: 0,18: 0))$, generally range between 
0.05-0.11 (Choi et al. 2000; Scollan et al. 2001a). The steers in the present study had a high muscle fat content $(6600 \mathrm{~g}$ fatty acids/ $100 \mathrm{~g}$ muscle), and so the low P:S ratio results were consistent with this negative exponential relationship.

Replacing lard with FO in the diets of finishing cattle had a beneficial effect on the $n-6: n-3$ PUFA ratio of intramuscular fat. The ratio observed was below 4:1, a value regarded as compliant with the guidelines from the Department of Health (1994). In the present study, the addition of FO contributed to a modification of the proportion of long-chain $n-3$ and $n$-6 fatty acids, without affecting $18: 2 n-6$ and $18: 3 n-3$, leading to a reduction in the $n-6: n-3$ PUFA ratio to $2 \cdot 95: 1$ (in steers fed $40 \mathrm{~g} \mathrm{FO} / \mathrm{kg}$ ). Based on the data from this study, and assuming an average beef consumption of $100 \mathrm{~g} / \mathrm{day}$ (Enser et al. 1996), beef produced using the $40 \mathrm{~g} \mathrm{FO} / \mathrm{kg}$ concentrate (averaged across the type of silage) would supply $67 \mathrm{mg}$ of $n$-3 PUFA (36 mg of which come from $n-3$ long-chain PUFA), accounting for $20-40 \%$ of the recommended daily intake of $n-3$ fatty acids (Department of Health, 1994).

\section{Conclusions}

Notwithstanding the fact that emerging data on the human health benefits of CLA are not as dramatic as those from model systems and concerns about the consumption of trans PUFA, the results of this experiment are interpreted as indicating that inclusion of FO in rations used for beef cattle has a positive impact on the fatty acid profile of muscle and subcutaneous adipose tissue lipids from a human health perspective. The positive linear relationship between the level of inclusion of FO in the ration and the level of beneficial fatty acids in tissue offers opportunities for inclusion of higher levels of FO. The study also demonstrated that utilisation of wilted rather than unwilted silage will enhance the CLA content of bovine intramuscular fat and subcutaneous adipose tissue without a negative effect on the incorporation of $n-3$ PUFA.

\section{Acknowledgements}

This research was supported through the European Union, Fifth Framework Programme (Project QLRT-2000-31 423, 'Healthy Beef'). The technical assistance of V. McHugh, A. McArthur, N. Blount (Grange Research Centre) and D. Donovan (Moorepark Research Centre) is gratefully acknowledged, as is the cooperation of Meadow Meats, Rathdowney, Ireland.

\section{References}

AbuGhazaleh AA, Schingoethe DJ, Hippen AR \& Whitlock LA (2002) Feeding fish meal and extruded soybeans enhances the conjugated linoleic acid (CLA) content of milk. J Dairy Sci 85, $624-631$.

AbuGhazaleh AA, Schingoethe DJ, Hippen AR \& Kalscheur KF (2003) Milk conjugated linoleic acid response to fish oil supplementation of diets differing in fatty acid profiles. J Dairy Sci 86, 944-953.

Association of Analytical Chemists (1990) Official Methods of Analysis, 15th ed. Washington, DC: Association of Analytical Chemists.
Ashes JR, Siebert BD, Gulati SK, Cuthbertson AZ \& Scott TW (1992) Incorporation of $n-3$ fatty acids of fish oil into tissue and serum lipids of ruminants. Lipids 27, 629-631.

Boufaïed H, Chouinard PY, Tremblay GF, Petit HV, Michaud R \& Belanger G (2003) Fatty acids in forages I. Factors affecting concentrations. Can J Anim Sci 83, 501-511.

Chilliard Y, Ferlay A \& Doreau M (2001) Effect of different types of forages, animal fat or marine oils in cow's diet on milk fat secretion and composition, especially conjugated linoleic acid (CLA) and polyunsaturated fatty acids. Livest Prod Sci 70, 31-48.

Chin SF, Liu W, Storkson JM, Ha YL \& Pariza MW (1992) Dietary sources of conjugated linoleic acid, a newly recognised class of anticarcinogens. J Food Compos Anal 5, 185-197.

Choi NJ, Enser M, Wood JD \& Scollan ND (2000) Effect of breed on the deposition in beef muscle and adipose tissue of dietary $n-3$ polyunsaturated fatty acids. Anim Sci 71, 509-519.

De la Torre A, Debiton E, Juaneda P, Durand D, Chardigny JM, Baerthomeuf C, Bauchart D \& Gruffat D (2006) Beef conjugated linoleic acid isomers reduce human cancer cell growth even when associated with other beef fatty acids. $B r J$ Nutr 95, 346-352.

Demeyer D \& Doreau M (1999) Targets and procedures for altering ruminant milk and meat lipids. Proc Nutr Soc 58, 593-607.

Department of Health (1994) Report on Health and Social Subjects No. 46. Nutritional Aspects of Cardiovascular Disease. London: H.M. Stationery Office.

Dewhurst RJ \& King PJ (1998) Effect of extended wilting, shading and chemical additives on the fatty acids in laboratory grass silages. Grass Forage Sci 53, 219-224.

European Communities (1984) European Communities (Marketing of Feedstuffs) Regulation. Statutory Instruments SI No. 200 of 1984.

Enser M, Hallett K, Hewett B, Fursey GAF \& Wood JD (1996) Fatty acid content and composition of English beef, lamb and pork at retail. Meat Sci 42, 443-456.

Enser M, Scollan ND, Choi NJ, Kurt E, Hallett K \& Wood JD (1999) Effect of dietary lipid on the content of conjugated linoleic acid (CLA) in beef muscle. Anim Sci 69, 143-146.

Griinari JM, Corl BA, Lacy SH, Chouinard PY, Nurmela KVV \& Bauman DE (2000) Conjugated linoleic acid is synthesized endogenously in lactating dairy cows by $\Delta^{9}$-desaturase. $J$ Nutr 130, 2285-2291.

Gulati SK, Ashes JR \& Scott TW (1999) Hydrogenation of eicosapentaenoic and docosahexaenoic acids and their incorporation into milk fat. Anim Feed Sci Technol 79, 57-64.

Ha YL, Grimm NL \& Pariza MW (1987) Anticarcinogens from fried ground beef: heat altered derivatives of linoleic acid. Carcinogenesis 8, 1881-1887.

Kepler CR \& Tove SB (1967) Biohydrogenation of unsaturated fatty acid. J Biol Chem 242, 5686-5692.

Kuhnt K, Kraft J, Moeckel P \& Jahreis G (2006) Trans-11-18:1 is effectively delta9-desaturated in humans. Br J Nutr 95, $752-761$.

Jakobsen MU (2006) Intake of ruminant versus industrially produced trans fatty acids and the risk of coronory heart disease - what is the epidemiological evidence? Proceedings of the 4th European Federation Lipid Congress, p. 18.

Lee MRF, Tweed JKS, Moloney AP \& Scollan ND (2005) The effects of fish oil supplementation on rumen metabolism and the biohydrogenation of unsaturated fatty acids in beef steers given diets containing sunflower oil. Anim Sci 80, 361-367.

Mandell IB, Buchanan-Smith JG, Holub BJ \& Campbell CP (1997) Effects of fish meal in beef cattle diets on growth performance, carcass characteristics, and fatty acid composition of Longissimus muscle. J Anim Sci 75, 910-919.

Martin SA \& Jenkins TC (2002) Factors affecting conjugated linoleic acid and trans- $\mathrm{C}_{18: 1}$ fatty acid production by mixed ruminal bacteria. J Anim Sci 80, 3347-3352. 
Mir PS, Mir Z, Huber PS, et al. (2002) Growth, carcass characteristics, muscle conjugated linoleic acid (CLA) content, and response to intravenous glucose challenge in high percentage Wagyu, Wagyu $\times$ Limousin and Limousin steers fed sunflower oil-containing diets. J Anim Sci 80, 2996-3004.

Mitchell GE, Reed AW \& Rogers SA (1991) Influence of feeding regimen on the sensory qualities and fatty acid composition of beef steaks. J Food Sci 56, 1102-1103.

Moloney AP, Read MP \& Keane MG (1996) Effects of ardacin supplementation on rumen fermentation and protein degradability in steers. Anim Feed Sci Technol 57, 97-110.

Morgan CA, Noble RC, Cocchi M \& McCartney R (1992) Manipulation of the fatty acid composition of pig meat lipids by dietary means. J Sci Food Agric 58, 357-368.

Noci F, Monahan FJ, French P \& Moloney AP (2005a) The fatty acid composition of muscle fat and subcutaneous adipose tissue of pasture-fed beef heifers: influence of the duration of grazing. J Anim Sci 83, 1167-1178.

Noci F, O'Kiely P, Monahan FJ, Stanton C \& Moloney AP (2005b) Conjugated linoleic acid concentration in $m$. longissimus dorsi from heifers offered sunflower oil based concentrates and conserved forages. Meat Sci 69, 509-518.

Offer NW, Speake BK, Dixon J \& Marsden M (2001) Effect of fish-oil supplementation on levels of $(n-3)$ polyunsaturated fatty acids in the lipoprotein fractions of bovine plasma. Anim Sci 73, 523-531.

Pariza MW, Park Y \& Cook ME (2001) The biologically active isomers of conjugated linoleic acid. Prog Lipid Res 40, 283-298.

Porter MG \& Murray RS (2001) The volatility of components of grass silage on oven drying and the inter-relationship between dry-matter content estimated by different analytical methods. Grass Forage Sci 56, 405-411.
Raes K, De Smet S \& Demeyer D (2004) Effect of dietary fatty acids on incorporation of long chain polyunsaturated fatty acids and conjugated linoleic acid in lamb, beef and pork meat: a review. Anim Feed Sci Technol 113, 199-221.

Scollan ND, Choi NJ, Kurt E, Fisher AV, Enser M \& Wood JD (2001a) Manipulating the fatty acid composition of muscle and adipose tissue in beef cattle. Br J Nutr 85, 115-124.

Scollan ND, Dhanoa MS, Choi NJ, Maeng WJ, Enser M \& Wood JD (2001b) Biohydrogenation and digestion of long chain fatty acids in steers fed on different samples of lipid. J Agric Sci 136, 345-355.

Scollan ND, Enser M, Gulati SK, Richardson I \& Wood JD (2003) Effects of including a ruminally protected lipid supplement in the diet on the fatty acid composition of beef muscle. Br J Nutr 90, 709-716.

Scollan N, Hocquette JF, Nuernberg K, Dannenberger D, Richardson I \& Moloney A (2006) Innovations in beef production systems that enhance the nutritional and health value of beef lipids and their relationship with meat quality. Meat Sci 74, $17-33$.

Shingfield KJ, Ahvenjärvi S, Toivonen V, Ärölä A, Nurmela KVV, Huhtanen P \& Griinari JM (2003) Effect of dietary fish oil on biohydrogenation of fatty acids and milk fatty acid content in cows. Anim Sci 77, 165-179.

Sukhija PS \& Palmquist DL (1988) Rapid method for determination of total fatty acid content and composition of feedstuffs and faeces. J Agric Food Chem 36, 1202-1206.

Yaqoob P, Tricon S, Burdge GC \& Calder PC (2006) Conjugated linoleic acids (CLAs) and health. In Improving the Fat Content of Foods, pp. 182-209 [C Williams and J Buttriss, editors]. Cambridge, UK: Woodhead Publishing Limited. 